
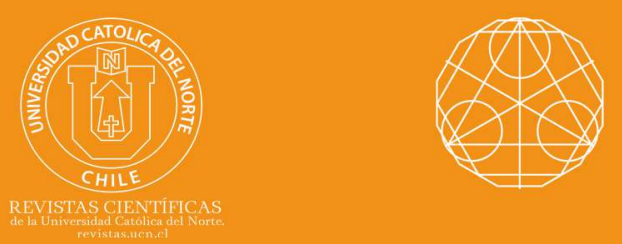

\title{
Maps preserving fixed points of generalized product of operators
}

Y. Bouramdane ${ }^{1}$ (1) orcid.org/0000-0002-4091-7206

M. Ech-Cherif El Kettani ${ }^{2}$

A. Elhiri ${ }^{3}$

A. Lahssaini ${ }^{4}$ @orcid.org/0000-0001-8114-170X

University Sidi Mohammed Ben Abdellah, Dept. of Mathematics, LASMA Laboratory, Fes, Morocco.

1 youssef.bouramdane1@usmba.ac.ma; ${ }^{2}$ mostapha.echcherifelkettani@usmba.ac.ma

3曰abdouhiri@gmail.com; ${ }^{4}$ aziz.lahssaini@usmba.ac.ma

Received: May 2019 | Accepted: July 2020

\section{Abstract:}

Let $\mathcal{B}(X)$ be the algebra of all bounded linear operators in a complex Banach space $X$. For $A \in \mathcal{B}(X)$ let $F(A)$ be the subspace of fixed point of $A$. For an integer $k \geq 2$, let $\left(i_{1}, . ., i_{m}\right)$ be a finite sequence with terms chosen from $\{1, \cdots, k\}$, and assume at least one of the terms in $\left(i_{1}, \cdots, i_{m}\right)$ appears exactly once. The generalized product of $k$ operators $A_{1}, \ldots, A_{k} \in \mathcal{B}(X)$ is defined by

$$
A_{1} * A_{2} * \cdots * A_{k}=A_{i_{1}} A_{i_{2}} \cdots A_{i_{m}},
$$

and includes the usual product and the triple product. We characterize the form of maps from $\mathcal{B}(X)$ onto itself satisfying

$$
F\left(\phi\left(A_{1}\right) * \cdots * \phi\left(A_{k}\right)\right)=F\left(A_{1} * \cdots * A_{k}\right)
$$

for all $A_{1}, \cdots, A_{k} \in \mathcal{B}(X)$.

Keywords: Fixed point; Generalized product; Preserver problems.

MSC (2020): 47B49,47B48, 47A10, 46H05.

\section{Cite this article as (IEEE citation style):}

Y. Bouramdane, M. Ech-Cherif El Kettani, A. Elhiri, and A. Lahssaini , "Maps preserving fixed points of generalized product of operators", Proyecciones (Antofagasta, On line), vol. 39, no. 5, pp. 1157-1165, Oct. 2020, doi: 10.22199/issn.0717-6279-202005-0071.

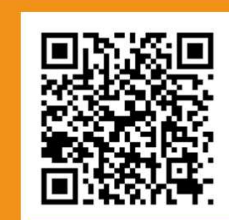

Article copyright: (C) 2020 Y. Bouramdane, M. Ech-Cherif El Kettani, A. Elhiri, and A. Lahssaini. This is an open access article distributed under the terms of the Creative Commons Licence, which permits unrestricted use and distribution provided the original author and source are credited. 


\section{Introduction}

Let $X$ be a Banach space over $\mathbf{K}$, where $\mathbf{K}$ is the complex field $\mathbf{C}$ or the real field $\mathbf{R}$, and let $\mathcal{B}(X)$ be the algebra of all bounded linear operators on $X$. The dual space of $X$ will be denoted by $X^{*}$. For a vector $x \in X$ and linear functional $f$ in the dual space $X^{*}$ of $X$, let $x \otimes f$ stands for the operator of rank at most one defined by

$$
(x \otimes f) y:=f(y) x, \quad(y \in X) .
$$

The problem of characterizing maps on matrices or operators that preserve certain functions, subsets and relations has attracted the attention of many mathematicians in the last decade; for example we can see $[1,2,3,4,5$, $6,7]$ and their references. In recent years, a great activity has occurred in characterising maps preserving the subspace of fixed points of a matrix or operators. Recall that a vector $x \in X$ is said to be fixed point of an operator $A \in \mathcal{B}(X)$ if $A x=x$, denote by $F(A)$ the set of all fixed points of an operator $A$. Note that if we consider the rank-one operator $x \otimes f$ for $x \in X$ and $f \in X^{*}$ then

$$
\begin{gathered}
x \otimes f \text { is idempotent } \Longleftrightarrow F(x \otimes f)=\langle x\rangle \\
x \otimes f \text { is not idempotent } \Longrightarrow F(x \otimes f)=\{0\} .
\end{gathered}
$$

Clearly that $F(A) \in \operatorname{Lat}(A)$, where $\operatorname{Lat}(A)$ the lattice of $A$, is the set of all invariant subspaces of $A$. In [4], A. A. Jafarian and A.R. Sourour described linear maps preserving the lattice of an operator in Banach alge- bra. In particular they showed that a linear map $\phi: \mathcal{B}(X) \rightarrow$ $\mathcal{B}(X)$ satisfied $\operatorname{Lat}(\phi(A))=\operatorname{Lat}(A)$, if and only if $\phi(A)=\alpha A+\varphi(A) I$ for all $A \in \mathcal{B}(X)$ where $I$ is the identity operator, $\alpha$ a nonzero scalar in $\mathbf{K}$ and $\varphi: \mathcal{B}(X) \rightarrow \mathbf{K}$ linear functional.

This result has been extended in [3], where G. Dolinar et al. characterised the form of maps preserving the lattice of sum of operators, they showed that maps (not necessarily linear) $\phi: \mathcal{B}(X) \rightarrow \mathcal{B}(X)$ satisfied $\operatorname{Lat}(\phi(A)+\phi(B))=\operatorname{Lat}(A+B)$ for all $A, B \in \mathcal{B}(X)$, if and only if there is a non zero scalar $\alpha$ and a map $\varphi: \mathcal{B}(X) \rightarrow \mathbf{K}$ such that $\phi(A)=\alpha A+\varphi(A) I$ for all $A \in \mathcal{B}(X)$. They proved also, in the same paper, that a non necessarily linear maps $\phi: \mathcal{B}(X) \rightarrow \mathcal{B}(X)$ satisfied $\operatorname{Lat}(\phi(A) \phi(B))=\operatorname{Lat}(A B)($ resp. $\operatorname{Lat}(\phi(A) \phi(B) \phi(A))=\operatorname{Lat}(A B A))$ for all $A, B \in \mathcal{B}(X)$, if and only if there is a map $\varphi: \mathcal{B}(X) \rightarrow \mathbf{K}$ such that $\varphi(A)=0$ if $A=0$ and $\phi(A)=\varphi(A) A$ for all $A \in \mathcal{B}(X)$.In [5], A. Taghavi 
and $\mathrm{R}$. Hosseinzadeh proved that if $X$ is a complex Banach space with $\operatorname{dim} X \geq 3$ and if a surjective map $\phi: \mathcal{B}(X) \rightarrow \mathcal{B}(X)$ satisfies

$$
\operatorname{dim} F(\phi(A) \phi(B))=\operatorname{dim} F(A B)
$$

for all $A, B \in \mathcal{B}(X)$, then there exists an invertible operator $S \in \mathcal{B}(X)$ such that $\phi(A)= \pm S A S^{-1}$ for all $A \in \mathcal{B}(X)$. In [6] A. Taghavi et al. studied the surjective maps $\phi: \mathcal{B}(X) \rightarrow \mathcal{B}(X)$ which satisfy $F(\phi(A)+\phi(B))$ $=F(A+B)$ for all $A, B \in \mathcal{B}(X)$, they conclude that $\phi(A)=U A+R$ for all $A \in \mathcal{B}(X)$ where $U=I-2 \phi(0)$ and $R=\phi(0)$. In [7] A. Taghavi et al. proved that if $X$ is a complex Banach space with $\operatorname{dim} X \geq 3$ and $\phi: \mathcal{B}(X)$ $\rightarrow \mathcal{B}(X)$ is sur- jective maps satifying $F(\phi(A) \phi(B) \phi(A))=F(A B A)$ for all $A, B \in \mathcal{B}(X)$, then $\phi(A)=\alpha A$ for all $A \in \mathcal{B}(X)$ where $\alpha \in \mathbf{C}$ with $\alpha^{3}=1$. The aim of this note is to prove the last result for the generalized product.

For an integer $k \geq 2$, let $\left(i_{1}, \cdots, i_{m}\right)$ be a finite sequence such that $\left\{i_{1}, \cdots, i_{m}\right\}=\{1 \cdots k\}$ and let at least one of the terms in $\left(i_{1}, \cdots, i_{m}\right)$ appears exactly once. The generalized product of width $m$ of $k$ operators $A_{1} \cdots A_{k} \in \mathcal{B}(X)$ is defined by

$$
A_{1} * A_{2} * \cdots * A_{k}=A_{i_{1}} A_{i_{2}} \cdots A_{i_{m}} .
$$

Evidently, the generalized product includes the usual product and the triple product. The following theorem is the main result of this paper. Its proof use ideas from $[1,7]$.

Theorem 1.1. Consider the generalized product of width $m, T_{1} * \cdots * T_{k}$. Let $\phi: \mathcal{B}(X) \rightarrow \mathcal{B}(X)$ be a surjective map. Then, the following statements are equivalent.

1. $F\left(\phi\left(A_{1}\right) * \cdots * \phi\left(A_{k}\right)\right)=F\left(A_{1} * \cdots * A_{k}\right)$ for all $A_{1}, \cdots, A_{k} \in \mathcal{B}(X)$.

2. There exists $\alpha \in \mathbf{C}$ with $\alpha^{m}=1$ such that $\phi(A)=\alpha A$ for all $A \in$ $\mathcal{B}(X)$.

\section{Preliminaries}

In this section, we collect and prove some lemmas that will be used in the sequel. The first and the second are quoted from [7]. Denote $C^{*}=$ $\mathbf{C} \backslash\{0,1\}$. Lemma 2.1. Let $A \in \mathcal{B}(X)$, then $A \in \mathbf{C}^{*} I$ if and only if $F(P A P)=\{0\}$, for every rank one idempotent operators $P \in \mathcal{B}(X)$. 
Proof. See [7, Lemma 2.1].

Lemma 2.2. Let $A$ and $B$ be non-scalar operators. If $F(P A P)=F(P B P)$, for every rank one idempotent operators $P \in \mathcal{B}(X)$, then there exists a scalar $\lambda \in \mathbf{C} \backslash\{1\}$ such that $B=\lambda I+(1-\lambda) A$.

Proof. See [7, Lemma 2.2].

In the following we give a conditions in term of dimension of fixed points of generalized product for two operators to be the same.

Lemma 2.3. Let $A$ and $B$ in $\mathcal{B}(X) \backslash\{0\}$, and $r$ and $s$ two positive integers such that $r+s \geq 1$. The following statements are equivalent.

1. $A=B$.

2. $\operatorname{dim} F\left(T^{r} A T^{s}\right)=\operatorname{dim} F\left(T^{r} B T^{s}\right)$ for all operators $T \in \mathcal{B}(X)$.

3. $\operatorname{dim} F\left(R^{r} A R^{s}\right)=\operatorname{dim} F\left(R^{r} B R^{s}\right)$ for all rank one operators $R \in \mathcal{B}(X)$.

Proof. The implications $(i) \Rightarrow($ ii $) \Rightarrow$ (iii) can be easily obtained. It remains to show that $(i i i) \Rightarrow(i)$. So, by Lemma 2.1, it is clear that $A \in \mathbf{C}^{*} I$ if and only if $B \in \mathbf{C}^{*} I$. Let $A=\alpha I$ and $B=\beta I$, for some $\alpha, \beta \in \mathbf{C}^{*}$. By assumption, we have

$$
\operatorname{dim} F\left(f(x)^{r+s-2} f(A x) x \otimes f\right)=\operatorname{dim} F\left(f(x)^{r+s-2} f(B x) x \otimes f\right)
$$

which implies that $f(x)^{r+s}=\alpha^{-1}$ if only if $f(x)^{r+s}=\beta^{-1}$ and so $\alpha=\beta$. Now let $A$ be a non-scalar operator. Since $B$ is a non-scalar operator too, we can apply Lemma 2.2. Thus, there exists a scalar $\lambda \in \mathbf{C} \backslash\{1\}$ such that $B=\lambda I+(1-\lambda) A$. It is enough to prove that $\lambda=0$. Assume on the contrary that $\lambda=0$ and let $x \in X$ and $f \in X^{*}$ such that $f(x)=, 1,-1$ and $f(A x)=\frac{1}{f(x)^{r+s-1}}$. It is clear that

$$
\operatorname{dim}\left((x \otimes f)^{r} A(x \otimes f)^{s}\right)=1 .
$$

Also we obtain

$$
(x \otimes f)^{r}(\lambda I+(\lambda) A)(x \otimes f)^{s}=f(x)^{r+s-1}(\lambda f(x)+(1-\lambda) f(A x))=1
$$

which implies that

$$
\operatorname{dim} F(x \otimes f)^{r}(\lambda I+(\lambda) A)(x \otimes f)^{s}=\operatorname{dim} F\left((x \otimes f)^{r} B(x \otimes f)^{s}\right)=0 .
$$


This is a contradiction. It follows that $A=B$ and thus the lemma is established.

Finally, we close this section with the following lemma that gives a characterization of rank-one operators by the dimension of fixed points of generalized product of operators.

Lemma 2.4. Let $r$ and $s$ be positive integers such that $r+s \geq 1$. For a nonzero operator $R \in \mathcal{B}(X)$, the following statements are equivalent.

1. $R$ is a rank one operator.

2. $\left.\operatorname{dim} F\left(T^{r} R T^{s}\right)\right) \leq 1$ for all $T \in \mathcal{B}(X)$.

Proof. If $R$ is a rank one operator and $T \in \mathcal{B}(X)$ is an arbitrary operator, then $T^{r} R T^{s}$ has rank at most one. Therefore, by (1.1) and (1.2), we have $\operatorname{dim} F\left(T^{r} R T^{s}\right) \leq 1$, and the implication $(i) \Rightarrow(i i)$ is established. Conversely, assume that $R$ has rank at least two. Let us show that there exists $T \in \mathcal{B}(X)$ such that $\operatorname{dim} F\left(T^{r} R T^{s}\right) \geq 2$. Since $\operatorname{rank}(R) \geq 2$, let $y_{1}$, $y_{2}$ be two linearly independent vectors in the range of $R$, and $x_{1}, x_{2}$ in $X$ such that $R x_{1}=y_{1}$ and $R x_{2}=y_{2}$. Assume that $s \neq 0$ and $r \neq 0$. Since $X$ has infinite dimension, we can find linearly independent vectors

$$
z_{0}, z_{1}, \ldots, z_{s}, z_{s+1}, \ldots, z_{s+r-1}, w_{0}, w_{1}, \ldots, w_{s}, \ldots, w_{s+r-1}
$$

with $z_{s}:=y_{1}$ and $w_{s}:=y_{2}$. Now, we can find a finite rank operator $T \in \mathcal{B}(X)$ such that

$$
T z_{s-1}=x_{1}, T z_{s+r-1}=z_{0}, T w_{s-1}=x_{2}, T w_{s+r-1}=w_{0}
$$

and for $i \in\{0,1, \ldots, r+s-1\} \backslash\{s-1, r+s-1\}$

$$
T z_{i}=z_{i+1} \text { and } T w_{i}=w_{i+1} .
$$

Note that $T^{r} R T^{s} z_{0}=z_{0}$ and $T^{r} R T^{s} w_{0}=w_{0}$. Then $z_{0}, w_{0} \in F\left(T^{r} R T^{s}\right)$, hence $\operatorname{dim} F\left(T^{r} R T^{s}\right) \geq 2$. This shows that the implication $(i i) \Rightarrow(i)$ always holds in case both $r$ and $s$ are positive integers.

To finish, we may and will assume that $s=0$ as the case when $r=0$ is similar. Then choose linearly independent vectors $z_{0}, z_{1}, \ldots, z_{r-1}, w_{0}, w_{1}, \ldots, w_{r-1}$ in $X$ with $z_{0}=y_{1}, w_{0}=y_{2}$. Thus, there exists a finite rank operator $T \in \mathcal{B}(X)$ such that $T z_{i}=z_{i+1}$ for $i \in\{0, r-2\}$ and $T z_{r-1}=x_{1}$ and $T w_{i}=w_{i+1}$ for $i \in\{0, r-2\}$ and $T w_{r-1}=x_{2}$. We have $T^{r} R x_{1}=x_{1}$ and $T^{r} R x_{2}=x_{2}$. Hence, $x_{1}, x_{2} \in F\left(T^{r} R\right)$ and $\left.\operatorname{dim} F\left(T^{r} R\right)\right) \geq 2$. This establishes the implication in this case too and the proof is then complete. 


\section{Main Results}

Since all the necessary ingredients are collected in the preliminary section we will state and prove the promised main result. Let $A, B \in \mathcal{B}(X)$, set $A_{i_{p}}=B$ and $A_{i_{j}}=A$ for $j \neq p$ with $i_{p}$ is the term which appears exactly once in $\left(i_{1}, \cdots, i_{m}\right)$. Note that $A_{1} * A_{2} * \cdots * A_{k}=A^{r} B A^{s}$ for some positive integers $r$ and $s$ such that $r+s=m-1$. Then, the Theorem 1.1 is a consequence of the following one.

\section{Theorem 3.1.}

Let $r$ and $s$ be two positive integers with $r+s \geq 1$ and $\phi: \mathcal{B}(X) \rightarrow$ $\mathcal{B}(X)$ be a surjective map. Then the following statements are equivalent.

1. For all $A, B \in \mathcal{B}(X)$,

$$
F\left(\phi(A)^{r} \phi(B) \phi(A)^{s}\right)=F\left(A^{r} B A^{s}\right) .
$$

2. There exists $\alpha \in \mathbf{C}$ with $\alpha^{r+s+1}=1$ such that $\phi(A)=\alpha A$ for all $A \in \mathcal{B}(X)$.

Proof. The 'if' part is easily verified, so we need only to prove the 'only if' part. Indeed, assume that $\phi$ is a surjective map from $\mathcal{B}(X)$ into itself such that for all $A, B \in \mathcal{B}(X)$,

$$
F\left(\phi(A)^{r} \phi(B) \phi(A)^{s}\right)=F\left(A^{r} B A^{s}\right) .
$$

We divide the proof into several steps.

Step 1. $\phi(0)=0$ and $\phi$ is injective.

By assumption, $\phi$ is surjective, so there exist $A \in \mathcal{B}(X)$ such that $\phi(A)=$ $x \otimes f$. Then by hypothesis we have

$$
\begin{aligned}
\{0\} & =F\left(A^{r} 0 A^{s}\right) \\
& =F\left(\phi(A)^{r} \phi(0) \phi(A)^{s}\right) \\
& =F\left((x \otimes f)^{r} \phi(0)(x \otimes f)^{s}\right) \\
& =F\left(f(x)^{r+s-2} f(\phi(0) x) x \otimes f\right)
\end{aligned}
$$

which implies that

$$
f(x)^{r+s-1} f(\phi(0) x) \neq 1 .
$$

So $x$ and $\phi(0) x$ are linearly dependant for every $x \in X$, because otherwise, we can find a linear functional $f$ such that $f(x) \neq 0$ and $f(\phi(0) x)=$ 
$\frac{1}{f(x)^{r+s-1}}$. Thus, there exists a complex number $\gamma$ such that $\phi(0)=\gamma I$. Replace in 3.2, we get $\gamma f(x)^{r+s-1} \neq 1$. Hence $\gamma=0$ and $\phi(0)=0$.

Let $A, B \in \mathcal{B}(X)$ such that $\phi(A)=\phi(B)$. Then, by hypothesis, for every $R \in \mathcal{B}(X)$ we have

$$
\begin{aligned}
F\left(R^{r} A R^{s}\right) & =F\left(\phi(R)^{r} \phi(A) \phi(R)^{s}\right) \\
& =F\left(\phi(R)^{r} \phi(B) \phi(R)^{s}\right) \\
& =F\left(R^{r} B R^{s}\right) .
\end{aligned}
$$

It follows, by lemma 2.3 that $A=B$, and so $\phi$ is injective. Thus $\phi$ is bijective since it is assumed surjective, moreover $\phi^{-1}$ satisfied the equation (3.1).

Step 2. $\phi$ preserves rank one operators in both directions.

This is obvious by Lemma 2.4 and the assumption that $\phi$ is surjective.

Step 3. $\phi(P)=\alpha P$ for every rank-one idempotent $P$ and some scalar $\alpha \in \mathbf{C}$ with $\alpha^{r+s+1}=1$.

Let $P \in \mathcal{P}_{1}(X)$ be a rank-one idempotent operator. Note $P=x \otimes f$ where $x \in X$ and $f \in X^{*}$ with $f(x)=1$. By the previous step $\phi$ preserves rankone operators in both directions. Then there exist $y \in X$ and $g \in X^{*}$ such that $\phi(P)=y \otimes g$. From 3.1 and hypothesis, we have

$$
\begin{aligned}
\langle x\rangle=F(P) & =F\left(P^{r} P P^{s}\right) \\
& =F\left(\phi(P)^{r} \phi(P) \phi(P)^{s}\right) \\
& =F\left(f(y)^{r+s} y \otimes g\right) .
\end{aligned}
$$

Then $\langle x\rangle=\langle y\rangle$ and so $x$ and $y$ are linearly dependant. Without loss of generality we can assume that $x=y$ and so $\phi(x \otimes f)=x \otimes g$. By (3.3) we obtain

$$
g(x)^{r+s+1}=1
$$

and so $x \notin \operatorname{ker}(g)$. Thus we have

$$
\operatorname{ker}(f) \cup\langle x\rangle=X, \operatorname{ker}(g) \cup\langle x\rangle=X
$$

and its clear that

$$
\operatorname{ker}(f) \cap\langle x\rangle=\{0\}, \operatorname{ker}(g) \cap\langle x\rangle=\{0\}
$$


which imply that $\operatorname{ker}(f)=\operatorname{ker}(g)$ and so $f$ and $g$ are linearly dependant. This and (3.4) yield that there exists a complex number $\alpha$ with $\alpha^{r+s+1}=1$ such that $\phi(P)=\alpha P$.

Step 4. $\phi(A)=\alpha A$ for every $A \in \mathcal{B}(X)$ and some scalar $\alpha \in \mathbf{C}$ with $\alpha^{r+s+1}=1$.

If $A$ is a rank one operator, by Lemma 2.2 there exists a $\lambda \in \mathbf{C} \backslash\{1\}$ such that

$$
\phi(A)=\alpha(\lambda I+(1-\lambda) A)
$$

because by Lemma 2.1, $A$ and $\phi(A)$ are non-scalar operators and by Step $3, \phi(P)=\alpha P$ for every rank one idempotent $P$. Since $\phi(A)$ is a rank one operator, we obtain $\lambda=0$ and so $\phi(A)=\alpha A$ for every rank one operator $A$. Now if $A$ is an arbitrary operator, the assertion follows from Lemma 2.4 , then $\phi$ takes the desired form.

By taking $r=s=1$ the main theorem of [7] becomes corollary of Theorem 1.1.

\section{Corollary 3.2.}

Let $\phi: \mathcal{B}(X) \rightarrow \mathcal{B}(X)$ be a surjective map. Then the following statements are equivalent.

1. $F(\phi(A) \phi(B) \phi(A))=F(A B A)$ for all $A, B \in \mathcal{B}(X)$.

2. There exists $\alpha \in \mathbf{C}$ with $\alpha^{3}=1$ such that $\phi(A)=\alpha A$ for all $A \in$ $\mathcal{B}(X)$.

\section{References}

[1] Z. E. A. Abdelali, A. Achchi, and R. Marzouki, "Maps preserving the local spectral radius zero of generalized product of operators", Linear and multilinear algebra, vol. 67, no. 10, pp. 2021-2029, Jul. 2018, doi:10.1080/03081087.2018.1479371

[2] A. Achchi, "Maps preserving the inner local spectral radius zero of generalized product of operators", Rendiconti del Circolo Matematico di Palermo Series 2, vol. 68, pp. 355-362, 2019, doi: 10.1007/s12215-018-0363-9 
[3] G. Dolinar, S. Du, J. Hou, and P. Legiša, “General preservers of invariant subspace lattices", Linear algebra and its applications, vol. 429, no. 1, pp. 100-109, Jul. 2008, doi: 10.1016/j.laa.2008.02.007

[4] A. A. Jafarian and A. R. Sourour, "Linear maps that preserve the commutant, double commutant or the lattice of invariant subspaces", Linear and multilinear algebra, vol. 38, no. 1-2, pp. 117-129, Jul. 1994, doi: 10.1080/ 03081089508818345

[5] A. Taghavi and R. Hosseinzadeh, "Maps preserving the dimension of fixed points of products of operators", Linear and multilinear algebra, vol. 62, no. 10, pp. 1285-1292, Sep. 2013, doi: $10.1080 / 03081087.2013 .823680$

[6] A. Taghavi, R. Hosseinzadeh, and H. Rohi, "Maps preserving the fixed points of sum of operators", Operators and matrices, no. 3, pp. 563-569, 2015, doi: 10.7153/ oam-09-34

[7] A. Taghavi, R. Hosseinzadeh, and V. Darvish, "Maps preserving the fixed points of triple Jordan products of operators", Indagationes mathematicae, vol. 27, no. 3, pp. 850-854, Jun. 2016, doi: 10.1016/j.indag.2016.03.003 